\title{
CONVERGENCE OF LINEARIZED AND ADJOINT APPROXIMATIONS FOR DISCONTINUOUS SOLUTIONS OF CONSERVATION LAWS. PART 1: LINEARIZED APPROXIMATIONS AND LINEARIZED OUTPUT FUNCTIONALS*
}

\author{
MIKE GILES ${ }^{\dagger}$ AND STEFAN ULBRICH ${ }^{\ddagger}$
}

\begin{abstract}
This paper analyzes the convergence of discrete approximations to the linearized equations arising from an unsteady one-dimensional hyperbolic equation with a convex flux function. A simple modified Lax-Friedrichs discretization is used on a uniform grid, and a key point is that the numerical smoothing increases the number of points across the nonlinear discontinuity as the grid is refined. It is proved that this gives pointwise convergence almost everywhere for the solution to the linearized discrete equations with smooth initial data, and also convergence in the discrete approximation of linearized output functionals. In Part 2 [M. Giles and S. Ulbrich, SIAM J. Numer. Anal., 48 (2010), pp. 905-921] we extend the results to Dirac initial data for the linearized equation and will prove the pointwise convergence almost everywhere for the solution of the adjoint discrete equations. In addition, we present numerical results illustrating the asymptotic behavior which is analyzed.
\end{abstract}

Key words. conservation law, hyperbolic, linearized, adjoint, numerical analysis

AMS subject classifications. 65M12, 65M08

DOI. $10.1137 / 080727464$

\section{Introduction.}

1.1. Background. In recent years there has been considerable research in the computational fluid dynamics community into the development and use of adjoint equations for design optimization (e.g., [Jam95, AV99]), data assimilation (e.g., [CT87, TC87]), and error analysis (e.g., [BR01, BD03]). In almost every case, the adjoint equations have been formulated under the assumption that the original nonlinear flow solution is smooth. Since most applications have been for incompressible or subsonic flow, this has been valid; however, there is also considerable interest in transonic design applications for which there are shocks. The correct formulation and discretization of adjoint equations in the presence of shocks is therefore important, and that is the main motivation for the analysis in this paper.

The reason that shocks present a problem is that adjoint equations are defined to be adjoint to the equations obtained by linearizing the original nonlinear equations. Therefore, this raises the issue of linearized perturbations to the shock. A correct treatment of the inviscid analytic equations must linearize the shock jump equations which arise from conservation properties at the shock. However, for numerical approximations which rely on shock capturing, as opposed to shock fitting, it is not clear whether linearized shock capturing will yield the correct results.

*Received by the editors June 16, 2008; accepted for publication (in revised form) March 25, 2010; published electronically June 29, 2010.

http://www.siam.org/journals/sinum/48-3/72746.html

${ }^{\dagger}$ Mathematical Institute, University of Oxford, Oxford, OX1 3LB, United Kingdom (mike.giles@ maths.ox.ac.uk).

${ }_{\ddagger}^{\ddagger}$ Department of Mathematics, TU Darmstadt, 64289 Darmstadt, Germany (ulbrich@mathematik. tu-darmstadt.de). This author's research was supported by the DFG within the SFB 568 and the SPP 1253. 
The validity of linearized shock capturing for the particular application of shocks oscillating harmonically in flutter analysis was investigated by Lindquist and Giles [LG94]. Their numerical results demonstrated that shock capturing produces the correct prediction of integral quantities such as unsteady lift and moment provided the shock is spread over a number of grid points. It was argued, but not proved, that this is because the "viscous" shock profile remains invariant, to leading order, as the shock oscillates, and therefore the integral effect of the linearized shock motion is correct. As a result, linearized shock capturing is now the standard method of turbomachinery aeroelastic analysis [HCL94, SW98], even though there has been no proof of convergence of the numerical discretization.

There has been very little prior research into adjoint equations for flows with shocks. Giles and Pierce [GP01] have shown that the analytic derivation of the adjoint equations for the steady quasi-one-dimensional Euler equations requires the specification of an internal adjoint boundary condition at the shock. However, the numerical evidence [GP98] suggests that for this steady one-dimensional application the correct adjoint solution is obtained using either the "discrete" approach (in which one linearizes the discrete equations and then forms their adjoint) or the "continuous" approach (in which one discretizes the analytic adjoint equations). In the case of the discrete approach, this is due to the second order accuracy of conservative quasione-dimensional shock capturing [Gil96], whereas with the continuous approach it is thought to be because the use of numerical smoothing automatically selects the correct numerical solution which is smooth at the shock [GP98]. Homescu and Navon [HN03] and Bardos and Pironneau [BP03] have also addressed the correct formulation and approximation of adjoint equations in flows with shocks.

Ulbrich has derived the adjoint equations for one-dimensional conservation laws with source terms, and using the method of generalized characteristics he has analyzed the differentiability of objective functionals with respect to controls [Ulb02, Ulb03]. In these papers he proved that the correct formulation leads to an interior boundary condition for the adjoint equations along discontinuities in the original nonlinear solutions, which is automatically satisfied for so-called reversible solutions of the adjoint equation in the sense of [BJ98].

Looking at nonlinear hyperbolic equations with scalar conservation laws, there has been considerable prior research into the convergence of numerical approximations to the nonlinear equations. To mention just a few key papers, Crandall and Majda [CM80] proved convergence to the unique entropy solution for monotone difference approximations of scalar conservation laws, and Nessyahu and Tadmor [NT92] proved an optimal bound on the order of convergence for a certain class of numerical methods, using the Lip' norm introduced by Tadmor [Tad91].

Linearized conservation laws with discontinuous coefficients have been analyzed in [LeF90, BJ98]. The appropriate definition of measure valued solutions, as well as their existence and uniqueness, have been considered in [LeF90]. The definition of the measure valued solutions in [LeF90] is based on the averaged superposition of Volpert for functions of bounded variation to define the nonconservative product in the flux term. In this context, Volpert's definition is appropriate; a detailed study of more general definitions of nonconservative products is carried out in [DMLM95]. The solutions in [LeF90] coincide with the duality solutions considered in [BJ98] for general linear conservation laws with discontinuous coefficients satisfying a onesided Lipschitz condition. Moreover, [BJ98] also introduces reversible solutions for the backward problem of the corresponding nonconservative transport equation with discontinuous coefficients. 
Giles has obtained numerical results for the Burgers equation [Gil03], showing that numerical discretizations of the adjoint equations can converge to incorrect solutions as the grid is refined uniformly, unless the number of grid points across the discontinuity increases as the grid is refined. Using the discrete linearized scheme and the discrete adjoint scheme ensures automatically that the conservative linearized equations are discretized by a conservative scheme and the nonconservative adjoint equations by a nonconservative scheme. This avoids approximation errors, which are studied in detail by [HL94].

It is the desire for a theoretical understanding of these numerical results which has motivated the research in this present paper and in Part 2 [GU10]. We consider an unsteady one-dimensional hyperbolic equation with a convex scalar flux, such as the Burgers equation. We use a specific explicit discretization with a modified LaxFriedrichs flux with a smoothing coefficient which varies with grid resolution $h$ to increase the number of grid points across any discontinuity as $h \rightarrow 0$. Because the numerical discretization is monotone, for sufficiently small timesteps, the classical results of Crandall and Majda [CM80], as well as the more recent results of Nessyahu and Tadmor [NT92], prove convergence of the nonlinear discretization to the unique entropy solution. In this paper we prove that for initial data which is smooth apart from one or more discontinuities the corresponding linearized discretization yields solutions which converge pointwise to the analytic solution everywhere except along the discontinuities. Furthermore, it is proved that the discrete approximation of the linearized perturbation to output integrals converges to the analytic value. From this we will deduce in Part 2 [GU10] that the discrete adjoint approximation must converge to the analytic adjoint solution as $h \rightarrow 0$, everywhere except along two characteristics across which it is discontinuous. Furthermore, by an appropriate choice of the numerical smoothing, the order of convergence is $O\left(h^{\alpha}\right)$ for any $\alpha<1$.

1.2. The model problem. The model problem is the equation

$$
N(u) \equiv \frac{\partial u}{\partial t}+\frac{\partial f(u)}{\partial x}=0, \quad-\infty<x<\infty, \quad 0<t<T,
$$

in which $f(u)$ is a $C^{\infty}$ convex flux function with derivative $a(u)$.

Numerical results will be presented in Part 2 for initial data $u_{0}(x)$, which is continuous and leads to the formation of a single shock after a finite time. However, the numerical analysis in this paper will be performed for initial data with a single initial discontinuity at $x=0$, and with all derivatives in $(-\infty, 0)$ and $(0, \infty)$ having a finite $L_{1}$ norm. This condition implies that $u_{0}(x)$ has bounded variation, and hence $u(x, t)$ is bounded. An extension to much more general initial data will be presented in Part 2 [GU10].

The shock moves with a velocity given by the Rankine-Hugoniot jump relation

$$
\dot{x}_{s}[u]-[f]=0,
$$

where $[u] \equiv u\left(x_{s}^{+}, t\right)-u\left(x_{s}^{-}, t\right)$ denotes the jump in $u$ across the shock.

Linear perturbations to the inviscid solution due to perturbed initial conditions are governed by the linear PDE

$$
L(u) \widetilde{u} \equiv \frac{\partial \widetilde{u}}{\partial t}+\frac{\partial}{\partial x}(a \widetilde{u})=0 .
$$

The corresponding linearized perturbation to the shock position satisfies the ODE

$$
\dot{\widetilde{x}}_{s}[u]+\dot{x}_{s}\left[\widetilde{u}+\widetilde{x}_{s} \frac{\partial u}{\partial x}\right]-\left[a \widetilde{u}+\widetilde{x}_{s} \frac{\partial f}{\partial x}\right]=0 .
$$


Eliminating $\partial f / \partial x$ using (1.1) and noting that

$$
\frac{\mathrm{d}}{\mathrm{d} t}[u]=\left[\frac{\partial u}{\partial t}+\dot{x}_{s} \frac{\partial u}{\partial x}\right]
$$

one obtains

$$
\frac{\mathrm{d}}{\mathrm{d} t}\left(\widetilde{x}_{s}[u]\right)=\left[\left(a-\dot{x}_{s}\right) \widetilde{u}\right],
$$

subject to initial data $\widetilde{x}_{s}(0)=0$.

By integrating (1.3) and (1.4) it can be verified that if the initial data $\widetilde{u}_{0}(x)$ has compact support, then the quantity

$$
\int_{-\infty}^{x_{s}(t)} \widetilde{u}(x, t) \mathrm{d} x+\int_{x_{s}(t)}^{\infty} \widetilde{u}(x, t) \mathrm{d} x-\widetilde{x}_{s}(t)[u]_{t}
$$

is invariant in time. Here $[u]_{t}$ represents the jump in $u(x, t)$ across the shock at time $t$. At the final time $T$, if all of the characteristics from the compact support of $\widetilde{u}_{0}(x)$ have entered the shock, and so $\widetilde{u}(x, T)$ is zero on either side of the shock, it follows that

$$
-\widetilde{x}_{s}(T)[u]_{T}=\int_{-\infty}^{\infty} \widetilde{u}_{0}(x) \mathrm{d} x .
$$

Thus the final shock perturbation is proportional to the integral of the initial solution perturbation.

The linearized perturbations $\widetilde{u}$ and $\widetilde{x}_{s}$ can be used to analyze the linearized perturbations of output functionals. The output functional of interest is a tracking-type functional of the form

$J=\int_{-\infty}^{\infty} \gamma(x) G(u(x, T)) \mathrm{d} x=\int_{-\infty}^{x_{s}(T)} \gamma(x) G(u(x, T)) \mathrm{d} x+\int_{x_{s}(T)}^{\infty} \gamma(x) G(u(x, T)) \mathrm{d} x$,

where $\gamma$ is a weighting function with compact support and bounded variation. The corresponding linear perturbation is

$$
\begin{aligned}
\widetilde{J}= & \int_{-\infty}^{x_{s}(T)} \gamma(x) G^{\prime}(u(x, T)) \widetilde{u}(x, T) \mathrm{d} x+\int_{x_{s}(T)}^{\infty} \gamma(x) G^{\prime}(u(x, T)) \widetilde{u}(x, T) \mathrm{d} x \\
& -\widetilde{x}_{s}(T) \gamma\left(x_{s}(T)\right)[G]_{T}
\end{aligned}
$$

where $[G]_{T}$ represents the jump in $G(u(x, T))$ across the shock at the final time.

It can be shown [Ulb02, Ulb03, Gil03] that the adjoint formulation of the linearized functional perturbation is

$$
\widetilde{J}=\int_{0}^{1} w(x, 0) \widetilde{u}(x, 0) \mathrm{d} x,
$$

where $w(x, t)$ satisfies the adjoint PDE

$$
\frac{\partial w}{\partial t}+a \frac{\partial w}{\partial x}=0
$$


in the smooth regions on either side of the shock, with "initial" conditions at the final time,

$$
w(x, T)=\gamma(x) G^{\prime}(x, T),
$$

and along the shock the interior boundary condition

$$
w\left(x_{s}(t), t\right)=\gamma\left(x_{s}(T)\right)[G]_{T} /[u]_{T} .
$$

Note that it follows from this that the adjoint solution has the uniform constant value $[G]_{T} /[u]_{T}$ on all characteristics leading into the shock.

The central objective in this paper is to prove that under certain conditions a linearized discretization of a regularized PDE yields an approximation to the linear inviscid solution which is convergent pointwise everywhere except at the shock, and also gives a convergent approximation to the linearized functional $\widetilde{J}$.

In Part 2 [GU10] we will show that from this it will then follow that the solution to the corresponding adjoint discretization converges almost everywhere to the inviscid adjoint solution.

1.3. Numerical discretizations. The nonlinear equation is approximated on a mesh with uniform spacing $h$ and timestep $k$ by the finite difference equation

$$
k N_{j}\left(U_{j}^{n}\right) \equiv U_{j}^{n+1}-U_{j}^{n}+\frac{1}{2} r\left(f\left(U_{j+1}^{n}\right)-f\left(U_{j-1}^{n}\right)\right)-\varepsilon d\left(U_{j+1}^{n}-2 U_{j}^{n}+U_{j-1}^{n}\right)=0,
$$

with initial data $U_{j}^{0}=u_{0}\left(x_{j}\right)$, where

$$
r \equiv \frac{k}{h}, \quad d \equiv \frac{k}{h^{2}}, \quad F_{j}^{n} \equiv f\left(U_{j}^{n}\right),
$$

and $\varepsilon=h^{\alpha}$ for some constant $0<\alpha<1$. The inequality $\alpha>0$ ensures that the discretization is consistent when the analytic solution $u(x, t)$ is smooth. The inequality $\alpha<1$ ensures that the shock is spread over an increasing number of grid points as $h \rightarrow 0$. It will be proved that stability and monotonicity are achieved for sufficiently small $h$ by choosing $k$ such that $\varepsilon d=c$ for some positive constant $c<\frac{1}{2}$.

Linearizing the nonlinear discretization yields the following approximation of the linear PDE:

$$
\begin{aligned}
& k L_{j}\left(U_{j}^{n}\right) \widetilde{U}_{j}^{n} \\
& \equiv \widetilde{U}_{j}^{n+1}-\widetilde{U}_{j}^{n}+\frac{1}{2} r\left(a\left(U_{j+1}^{n}\right) \widetilde{U}_{j+1}^{n}-a\left(U_{j-1}^{n}\right) \widetilde{U}_{j-1}^{n}\right)-\varepsilon d\left(\widetilde{U}_{j+1}^{n}-2 \widetilde{U}_{j}^{n}+\widetilde{U}_{j-1}^{n}\right)
\end{aligned}
$$

$(1.7)=0$,

which is to be solved subject to initial data $\widetilde{U}_{j}^{0}=\widetilde{u}_{0}\left(x_{j}\right)$. Again, this is a consistent approximation if both $u(x, t)$ and $\widetilde{u}(x, t)$ are smooth. Note also that if $\widetilde{u}_{0}(x)$ has compact support, then summing over $j$ yields the result that

$$
\sum_{j=-\infty}^{\infty} \widetilde{U}_{j}^{n}=\text { const. }
$$

If the nonlinear tracking-type functional is approximated by trapezoidal integration, then the corresponding discretization of the linearized functional is

$$
\widetilde{J}_{h}=h \sum_{j} \gamma\left(x_{j}\right) G^{\prime}\left(U_{j}^{N}\right) \widetilde{U}_{j}^{N}
$$


where $N k=T$. The exactly equivalent adjoint formulation for this is

$$
\widetilde{J}_{h}=h \sum_{j} W_{j}^{0} \widetilde{U}_{j}^{0}
$$

where $W_{j}^{n}$ satisfies the discrete adjoint equation [Gil03]

$$
W_{j}^{n-1}=W_{j}^{n}+\frac{1}{2} r a\left(U_{j}^{n-1}\right)\left(W_{j+1}^{n}-W_{j-1}^{n}\right)+\varepsilon d\left(W_{j+1}^{n}-2 W_{j}^{n}+W_{j-1}^{n}\right),
$$

with "initial" conditions

$$
W_{j}^{N}=\gamma\left(x_{j}\right) G^{\prime}\left(U_{j}^{N}\right) .
$$

This adjoint formulation follows immediately from the identity

$$
\sum_{j} W_{j}^{n} \widetilde{U}_{j}^{n}=\sum_{j} W_{j}^{n+1} \widetilde{U}_{j}^{n+1}
$$

which is easily verified. In this paper we will prove that $\widetilde{J}_{h} \rightarrow \widetilde{J}$ as $h \rightarrow 0$ for smooth linear initial perturbations $\widetilde{u}_{0}$.

In the particular case of Dirac initial data for the linear discretization,

$$
\widetilde{U}_{j}^{0}=\left\{\begin{array}{cc}
h^{-1}, & j=J \\
0 & \text { otherwise }
\end{array}\right.
$$

then $\widetilde{J}_{h}=W_{J}^{0}$. Thus, the adjoint solution at a particular point is equal to the linear functional arising from Dirac initial data for the linearized equations at that same point. By proving in Part 2 [GU10] that the linearized functional converges to the correct value also for Dirac initial data, we will also be proving that the adjoint approximation converges to the analytic solution.

1.4. Outline of paper. This first paper is devoted to the proof of the convergence of the discrete linear functional $\widetilde{J}_{h}$ to the analytic value $\widetilde{J}$ as $h \rightarrow 0$. The convergence is analyzed by using the technique of matched inner and outer asymptotic expansions [BO78, KC81] to construct approximations to both $U_{j}^{N}$ and $\widetilde{U}_{j}^{n}$. Discrete stability estimates are used to bound the errors in the asymptotic approximations.

- Section 2 derives the stability estimates which are used later in section 4 to bound the errors in the asymptotic approximations.

- Section 3 derives the asymptotic form of the discrete approximation of a viscous traveling wave on a uniform grid, and then rescales this to obtain the asymptotic form of the discrete approximation to a moving shock with uniform conditions on either side.

- Section 4 uses the moving shock approximation to form blended inner/outer asymptotic approximations of both the nonlinear and linear discrete solutions for a particular choice of discrete initial data for the nonlinear and linearized equations. Together with the stability estimates, this proves the convergence of the linear solution away from the shock.

- Section 5 completes the main analysis by bounding the error in the linearized discrete functional approximation.

Part 2 [GU10] extends the analysis to linear problems with Dirac initial data, which implies the convergence of the discrete adjoint solution. Moreover, the results of this paper are extended to more general nonlinear initial data. 


\section{Discrete stability estimates.}

\subsection{Nonlinear equations.}

THEOREM 2.1. Suppose that $U_{j}^{n}$ is a solution of the equation

$$
U_{j}^{n+1}=U_{j}^{n}-\frac{1}{2} r\left(f\left(U_{j+1}^{n}\right)-f\left(U_{j-1}^{n}\right)\right)+\varepsilon d\left(U_{j+1}^{n}-2 U_{j}^{n}+U_{j-1}^{n}\right),
$$

where $f(u)$ is a $C^{\infty}$ function with derivative $a(u)=f^{\prime}(u)$, and $r=k / h, d=k / h^{2}$, $\varepsilon=h^{\alpha}, 0<\alpha<1$, and subject to specified initial data $U_{j}^{0}$ with $L_{\infty}$ bound $U_{\infty}$.

Furthermore, let $V_{j}^{n}$ be an approximation to $U_{j}^{n}$ which satisfies the equation

$$
V_{j}^{n+1}=V_{j}^{n}-\frac{1}{2} r\left(f\left(V_{j+1}^{n}\right)-f\left(V_{j-1}^{n}\right)\right)+\varepsilon d\left(V_{j+1}^{n}-2 V_{j}^{n}+V_{j-1}^{n}\right)+k \tau_{j}^{n},
$$

and the same initial data $U_{j}^{0}$, and let $U_{\infty}$ also be an upper bound on $\left\|V_{j}^{n}\right\|_{\infty}$.

Then, provided that $h<\left(2 / A_{\infty}\right)^{1 /(1-\alpha)}$ where $A_{\infty}=\sup _{|u|<U_{\infty}}|a(u)|$, and $k$ is chosen so that $\varepsilon d=c$ for some constant $c<\frac{1}{2}$, then $E_{j}^{n}=V_{j}^{n}-U_{j}^{n}$ satisfies the bound

$$
\left\|E^{n}\right\|_{1} \leq\|\tau\|_{1, n}
$$

where the $l_{1}$ norm is defined as $\left\|E^{n}\right\|_{1} \equiv h \sum_{j}\left|E_{j}^{n}\right|$ and $\|\tau\|_{1, n}=k \sum_{m=1}^{n}\left\|\tau^{m}\right\|_{1}$.

Proof. The conditions on $h$ and $k$ ensure that $\frac{1}{2} r A_{\infty}<c$. Hence, the equations for $U_{j}^{n}$ are monotone, and therefore $\left|U_{j}^{n}\right| \leq U_{\infty}$ and $\left|a\left(U_{j}^{n}\right)\right| \leq A_{\infty} \forall j, n$.

Defining

$$
A_{j}^{n}=\left\{\begin{array}{cc}
\frac{f\left(V_{j}^{n}\right)-f\left(U_{j}^{n}\right)}{V_{j}^{n}-U_{j}^{n}}, & V_{j}^{n} \neq U_{j}^{n}, \\
a\left(U_{j}^{n}\right), & V_{j}^{n}=U_{j}^{n},
\end{array}\right.
$$

the difference $E_{j}^{n}=V_{j}^{n}-U_{j}^{n}$ satisfies the equation

$$
E_{j}^{n+1}=E_{j}^{n}-\frac{1}{2} r\left(A_{j+1}^{n} E_{j+1}^{n}-A_{j-1}^{n} E_{j-1}^{n}\right)+c\left(E_{j+1}^{n}-2 E_{j}^{n}+E_{j-1}^{n}\right)+k \tau_{j}^{n},
$$

with homogeneous initial data. $A_{j}^{n}$ satisfies the bound $\left|A_{j}^{n}\right| \leq A_{\infty}$, and hence $\frac{1}{2} r\left|A_{j}^{n}\right|<c \forall j, n$.

If $\left\|\tau^{n}\right\|_{1}$ is finite, then taking the absolute magnitude and summing over the entire interval gives

$$
\sum_{j} h\left|E_{j}^{n+1}\right| \leq \sum_{j} h\left(\left|E_{j}^{n}\right|+k\left|\tau_{j}^{n}\right|\right)
$$

and hence $\left\|E^{n}\right\|_{1} \leq\|\tau\|_{1, n}$.

\subsection{Linearized equations.}

TheOREM 2.2. Given $U_{j}^{n}$ and $V_{j}^{n}$ as defined in Theorem 2.1 , let $\widetilde{U}_{j}^{n}$ be a solution of the linearized difference equation

$$
\widetilde{U}_{j}^{n+1}=\widetilde{U}_{j}^{n}-\frac{1}{2} r\left(a\left(U_{j+1}^{n}\right) \widetilde{U}_{j+1}^{n}-a\left(U_{j-1}^{n}\right) \widetilde{U}_{j-1}^{n}\right)+\varepsilon d\left(\widetilde{U}_{j+1}^{n}-2 \widetilde{U}_{j}^{n}+\widetilde{U}_{j-1}^{n}\right),
$$

and let $\widetilde{V}_{j}^{n}$ be an approximation to it which satisfies the equation

$\widetilde{V}_{j}^{n+1}=\widetilde{V}_{j}^{n}-\frac{1}{2} r\left(a\left(V_{j+1}^{n}\right) \widetilde{V}_{j+1}^{n}-a\left(V_{j-1}^{n}\right) \widetilde{V}_{j-1}^{n}\right)+\varepsilon d\left(\widetilde{V}_{j+1}^{n}-2 \widetilde{V}_{j}^{n}+\widetilde{V}_{j-1}^{n}\right)+k \widetilde{\tau}_{j}^{n}$,

with initial data $\widetilde{V}_{j}^{0}$ which may differ from $\widetilde{U}_{j}^{0}$. 
Then, provided $h$ and $k$ satisfy the same conditions as in Theorem 2.1, the difference $\widetilde{E}_{j}^{n}=\widetilde{V}_{j}^{n}-\widetilde{U}_{j}^{n}$ satisfies the bound

$$
\left\|\widetilde{E}^{n}\right\|_{1} \leq\left\|\widetilde{E}^{0}\right\|_{1}+h^{-1} t^{n} B_{\infty} \widetilde{V}_{\infty}\|\tau\|_{1, n}+\|\widetilde{\tau}\|_{1, n}
$$

where $B_{\infty}=\sup _{|u|<U_{\infty}}\left|a^{\prime}(u)\right|$, and $\widetilde{V}_{\infty}$ is an upper bound for $\left|\widetilde{V}_{j}^{n}\right|$.

Proof. Defining

$$
B_{j}^{n}=\left\{\begin{array}{cc}
\frac{a\left(V_{j}^{n}\right)-a\left(U_{j}^{n}\right)}{V_{j}^{n}-U_{j}^{n}}, & V_{j}^{n} \neq U_{j}^{n}, \\
a^{\prime}\left(U_{j}^{n}\right), & V_{j}^{n}=U_{j}^{n},
\end{array}\right.
$$

with bound $\left|B_{j}^{n}\right|<B_{\infty}$, the difference $\widetilde{E}_{j}^{n}=\widetilde{V}_{j}^{n}-\widetilde{U}_{j}^{n}$ satisfies the equation

$$
\begin{aligned}
\widetilde{E}_{j}^{n+1}= & \widetilde{E}_{j}^{n}-\frac{1}{2} r\left(a\left(U_{j+1}^{n}\right) \widetilde{E}_{j+1}^{n}-a\left(U_{j-1}^{n}\right) \widetilde{E}_{j-1}^{n}\right)+\varepsilon d\left(\widetilde{E}_{j+1}^{n}-2 \widetilde{E}_{j}^{n}+\widetilde{E}_{j-1}^{n}\right) \\
& -\frac{1}{2} r\left(B_{j+1}^{n} E_{j+1}^{n} \widetilde{V}_{j+1}^{n}-B_{j-1}^{n} E_{j-1}^{n} \widetilde{V}_{j-1}^{n}\right)+k \widetilde{\tau}_{j}^{n} .
\end{aligned}
$$

Taking the absolute magnitude, using the triangle inequality, and then summing over the entire interval gives

$$
\sum_{j} h\left|\widetilde{E}_{j}^{n+1}\right| \leq \sum_{j} h\left(\left|\widetilde{E}_{j}^{n}\right|+r B_{\infty}\left|\widetilde{V}_{j}^{n}\right|\left|E_{j}^{n}\right|+k\left|\widetilde{\tau}_{j}^{n}\right|\right) .
$$

Hence,

$$
\left\|\widetilde{E}^{n+1}\right\|_{1} \leq\left\|\widetilde{E}^{n}\right\|_{1}+r \quad B_{\infty} \widetilde{V}_{\infty}\|\tau\|_{1, n}+k\left\|\widetilde{\tau}^{n}\right\|_{1}
$$

and therefore

$$
\left\|\widetilde{E}^{n}\right\|_{1} \leq\left\|\widetilde{E}^{0}\right\|_{1}+h^{-1} t^{n} B_{\infty} \widetilde{V}_{\infty}\|\tau\|_{1, n}+\|\widetilde{\tau}\|_{1, n}
$$

\section{Analytic and discrete traveling wave solutions.}

3.1. Viscous traveling wave. We begin with a theorem which establishes the existence of a unique traveling wave solution to the viscous convection/diffusion equation with unit viscosity and a convex flux function.

TheOREM 3.1. Given the viscous equation

$$
\frac{\partial u}{\partial t}+\frac{\partial f}{\partial x}=\frac{\partial^{2} u}{\partial x^{2}}
$$

with a $C^{\infty}$ convex flux function $f(u)$ with derivative $a(u)$, then for any values of the constants $\dot{x}_{s}$ and $\Delta u$ satisfying the inequalities $\sup _{u} a(u)>\dot{x}_{s}>\inf _{u} a(u), \Delta u<0$, there exists a unique traveling wave solution of the form

$$
u(x, t)=s\left(\dot{x}_{s}, \Delta u ; x-\dot{x}_{s} t\right)
$$

with the properties that as $x^{*} \rightarrow \infty, s\left(-x^{*}\right) \rightarrow s_{-\infty}, s\left(x^{*}\right) \rightarrow s_{\infty}=s_{-\infty}+\Delta u$ for some constant $s_{-\infty}$, and

$$
\int_{-x^{*}}^{x^{*}} s(x) d x \rightarrow x^{*}\left(s_{-\infty}+s_{\infty}\right) .
$$

Furthermore, all derivatives of $s(x)$ decay exponentially as $|x| \rightarrow \infty$. 
Proof. Substituting the traveling wave ansatz into the PDE gives the equation

$$
-\dot{x}_{s} \frac{\mathrm{d} s}{\mathrm{~d} x}+\frac{\mathrm{d} f(s)}{\mathrm{d} x}=\frac{\mathrm{d}^{2} s}{\mathrm{~d} x^{2}}
$$

Integrating this yields

$$
f(s)-\dot{x}_{s} s-\frac{\mathrm{d} s}{\mathrm{~d} x}=C
$$

for some constant $C$. Given particular values for $\dot{x}_{s}$ and $\Delta u$ satisfying the specified inequalities, because of the convexity of $f(u)$ there exists a unique value $s_{-\infty}$ such that

$$
\dot{x}_{s}=\frac{f\left(s_{-\infty}+\Delta u\right)-f\left(s_{-\infty}\right)}{\Delta u} .
$$

Defining $s_{\infty}=s_{-\infty}+\Delta u$, and setting $C=f\left(s_{\infty}\right)-\dot{x}_{s} s_{\infty}$, gives $s(x)$ defined implicitly by

$$
\int_{s_{-\infty}+\frac{1}{2} \Delta u}^{s} \frac{\mathrm{d} u}{f(u)-\dot{x}_{s} u-C}=x-x_{0} .
$$

The quantity $f(u)-\dot{x}_{s} u-C$ is strictly negative for $s_{\infty}<u<s_{-\infty}$, because of the convexity of $f(u)$, and approaches zero linearly as $u \rightarrow s_{\infty}$ or $u \rightarrow s_{-\infty}$. Hence, all derivatives of $s(x)$ decay exponentially as $|x| \rightarrow \infty$.

Finally, the unique value of $x_{0}$ is determined by the requirement that, as $x^{*} \rightarrow \infty$,

$$
\int_{-x^{*}}^{x^{*}} s(x) \mathrm{d} x \rightarrow x^{*}\left(s_{-\infty}+s_{\infty}\right) .
$$

Next, we consider linear perturbations to (3.2), under the influence of a source term $g(x)$, giving the equation

$$
-\dot{x}_{s} \frac{\mathrm{d} \widetilde{s}}{\mathrm{~d} x}+\frac{\mathrm{d}}{\mathrm{d} x}(a(s) \widetilde{s})=\frac{\mathrm{d}^{2} \widetilde{s}}{\mathrm{~d} x^{2}}+g(x)
$$

TheOrem 3.2. When $g(x)=0$, all solutions of (3.4), subject to the boundary conditions $\widetilde{s}(x) \rightarrow 0$ as $|x| \rightarrow \infty$, are of the form

$$
\widetilde{s}=c \frac{d s}{d x}
$$

for some constant $c$.

When $g(x)$ is not identically zero, but $g(x)$ and its derivatives all decay exponentially as $|x| \rightarrow \infty$, there exist nonunique solutions of (3.4), subject to the boundary conditions $\widetilde{s}(x) \rightarrow \widetilde{s}_{\infty}$ and $\widetilde{s}(-x) \rightarrow \widetilde{s}_{-\infty}$ as $x \rightarrow \infty$, iff $g(x)$ satisfies the solvability condition

$$
\int_{-\infty}^{\infty} g(\xi) d \xi=\left(a\left(s_{\infty}\right)-\dot{x}_{s}\right) \widetilde{s}_{\infty}-\left(a\left(s_{-\infty}\right)-\dot{x}_{s}\right) \widetilde{s}_{-\infty}
$$

Furthermore, the derivatives of $\widetilde{s}(x)$ all decay exponentially as $|x| \rightarrow \infty$. 
Proof. Integrating (3.4) with $g(x)=0$ gives

$$
\left(a(s)-\dot{x}_{s}\right) \widetilde{s}=\frac{\mathrm{d} \widetilde{s}}{\mathrm{~d} x}+\text { const. }
$$

Because $a\left(s_{\infty}\right)-\dot{x}_{s}<0$ and $a\left(s_{-\infty}\right)-\dot{x}_{s}>0$, the boundary conditions are satisfied provided the integration constant is zero.

This equation can then be integrated subject to an arbitrary value of $\widetilde{s}(0)$ to give a one-parameter family of homogeneous solutions which satisfy the boundary conditions as $|x| \rightarrow \infty$.

Differentiating (3.2) establishes that $\mathrm{d} s / \mathrm{d} x$ satisfies the homogeneous version of (3.4) and the specified boundary conditions. Therefore, $\mathrm{d} s / \mathrm{d} x$ is a basis for the oneparameter family of homogeneous solutions with the specified boundary conditions.

When $g(x)$ is not identically zero, the solvability condition arises immediately from integrating (3.4) and applying the boundary conditions.

The exponential decay in the derivatives of $\widetilde{s}(x)$ follows from the exponential decay in $g(x)$ and its derivatives and also the exponential decay in the derivatives of $s(x)$.

3.2. Discrete traveling wave with unit viscosity. The objective in this section is to derive an approximation to the discrete traveling wave solution which arises when approximating (3.1) using the nonlinear discretization

$$
k N_{j}\left(U_{j}^{n}\right) \equiv U_{j}^{n+1}-U_{j}^{n}+\frac{1}{2} r\left(f\left(U_{j+1}^{n}\right)-f\left(U_{j-1}^{n}\right)\right)-d\left(U_{j+1}^{n}-2 U_{j}^{n}+U_{j-1}^{n}\right)=0,
$$

where $r=k / h$, and $d=k / h^{2}$ is held fixed as $h \rightarrow 0$.

THEOREM 3.3. For any values of the constants $\dot{x}_{s}$ and $\Delta u$ satisfying the conditions of Theorem 3.1, there exists a sequence of functions $c_{n}(x)$, with $c_{n}(x) \rightarrow 0$, as $|x| \rightarrow \infty$, such that for all integers $M \geq 0$ the function $S_{M}(x)$ defined by

$$
S_{M}(x)=s(x)+\sum_{n=1}^{M} h^{2 n} c_{n}(x)
$$

has the properties that

$$
N_{j}\left(S_{M}\left(x_{j}-\dot{x}_{s} t^{n}\right)\right)=o\left(h^{2 M}\right),
$$

and as $x^{*} \rightarrow \infty, S_{M}\left(-x^{*}\right) \rightarrow s_{-\infty}, S_{M}\left(x^{*}\right) \rightarrow s_{\infty}=s_{-\infty}+\Delta u$, and

$$
\int_{-x^{*}}^{x^{*}} S_{M}(x) d x \rightarrow x^{*}\left(s_{-\infty}+s_{\infty}\right) \text {. }
$$

Furthermore, all derivatives of $S_{M}(x)$ decay exponentially as $|x| \rightarrow \infty$.

Proof. The proof is by induction. Suppose that for a given $M \geq 0$ it is true that there exist functions $c_{n}(x)$ for $n \leq M$ such that $S_{M}(x)$ has the specified properties.

A truncated Taylor series expansion of $f(u)$ gives

$$
f(u)=f\left(u_{0}\right)+\left.\sum_{n=1}^{M+1} \frac{\left(u-u_{0}\right)^{n}}{n !} \frac{\mathrm{d}^{n} f}{\mathrm{~d} u^{n}}\right|_{u_{0}}+\frac{\left(u-u_{0}\right)^{M+2}}{(M+2) !} f_{M+2}\left(u_{0}, u\right)
$$

where

$$
f_{M+2}\left(u_{0}, u\right)=\left.\left(\int_{u_{0}}^{u}(u-\xi)^{M+1} \mathrm{~d} \xi\right)^{-1} \int_{u_{0}}^{u}(u-\xi)^{M+1} \frac{\mathrm{d}^{M+2} f}{\mathrm{~d} u^{M+2}}\right|_{\xi} \mathrm{d} \xi
$$

is a weighted average value of $\mathrm{d}^{M+2} f / \mathrm{d} u^{M+2}$ on the interval $\left[u_{0}, u\right]$. 
Using this expansion with $u_{0}=U_{j}^{n}=S_{M}\left(x_{j}-\dot{x}_{s} t^{n}\right)$ and $u=U_{j \pm 1}^{n}$, and then making similar truncated Taylor series expansions for $U_{j \pm 1}^{n}$ and $U_{j}^{n+1}$ (with $k=d h^{2}$ ), one finds that the residual error has an expansion in even powers of $h^{2}$ of the form

$$
N_{j}\left(S_{M}\left(x_{j}-\dot{x}_{s} t^{n}\right)\right)=\left.\sum_{n=1}^{M+1} h^{2 n} \frac{\mathrm{d} r_{n}}{\mathrm{~d} x}\right|_{x_{j}-\dot{x}_{s} t^{n}}+o\left(h^{2 M+2}\right),
$$

where $r_{n}(x) \rightarrow 0$, as $|x| \rightarrow \infty$. However, by the inductive hypothesis, this residual error is $o\left(h^{2 M}\right)$. Therefore,

$$
N_{j}\left(S_{M}\left(x_{j}-\dot{x}_{s} t^{n}\right)\right)=\left.h^{2 M+2} \frac{\mathrm{d} r_{M+1}}{\mathrm{~d} x}\right|_{x_{j}-\dot{x}_{s} t^{n}}+o\left(h^{2 M+2}\right) .
$$

Now, let $c_{M+1}\left(x-\dot{x}_{s} t\right)$ be defined by the linear differential equation

$$
L\left(s\left(x-\dot{x}_{s} t\right)\right) c_{M+1}\left(x-\dot{x}_{s} t\right)=-\left.\frac{\mathrm{d} r_{M+1}}{\mathrm{~d} x}\right|_{x-\dot{x}_{s} t},
$$

giving the $\mathrm{ODE}$

$$
-\dot{x}_{s} \frac{\mathrm{d} c_{M+1}}{\mathrm{~d} x}+\frac{\mathrm{d}}{\mathrm{d} x}\left(a(s(x)) c_{M+1}\right)-\frac{\mathrm{d}^{2} c_{M+1}}{\mathrm{~d} x^{2}}=-\frac{\mathrm{d} r_{M+1}}{\mathrm{~d} x},
$$

subject to the boundary conditions $c_{M+1}(x) \rightarrow 0$, as $|x| \rightarrow \infty$. Because the right-hand side of this equation satisfies the necessary solvability condition of Theorem 3.2, there exists a solution $c_{M+1}(x)$, and it is unique if we impose the additional constraint that

$$
\int_{-\infty}^{\infty} c_{M+1}(x) \mathrm{d} x=0
$$

Also, due to Theorem 3.2, all derivatives of $c_{M+1}$ decay exponentially as $|x| \rightarrow \infty$.

Finally, we obtain

$$
\begin{aligned}
N_{j} & \left(S_{M}\left(x_{j}-\dot{x}_{s} t^{n}\right)+h^{2 M+2} c_{M+1}\left(x_{j}-\dot{x}_{s} t^{n}\right)\right) \\
& =N_{j}\left(S_{M}\left(x_{j}-\dot{x}_{s} t^{n}\right)\right)+h^{2 M+2} L_{j}\left(U_{j}^{n}\right) c_{M+1}\left(x_{j}-\dot{x}_{s} t^{n}\right)+o\left(h^{2 M+2}\right) \\
& =N_{j}\left(S_{M}\left(x_{j}-\dot{x}_{s} t^{n}\right)\right)+\left.h^{2 M+2} L_{s\left(x-\dot{x}_{s} t\right)} c_{M+1}\right|_{x_{j}-\dot{x}_{s} t^{n}}+o\left(h^{2 M+2}\right) \\
& =o\left(h^{2 M+2}\right),
\end{aligned}
$$

and

$$
\int_{-x^{*}}^{x^{*}} S_{M}(x)+h^{2 M+2} c_{M+1}(x) \mathrm{d} x \longrightarrow \int_{-x^{*}}^{x^{*}} S_{M}(x) \mathrm{d} x, \quad \text { as } x^{*} \rightarrow \infty,
$$

so the inductive hypothesis is true for $M+1$. The hypothesis is trivially true for the initial value $M=0$, concluding the proof.

Although the above proof has used the shorthand $S_{M}(x)$, we should more properly express it as $S_{M}\left(d, h, \dot{x}_{s}, \Delta u ; x\right)$ to make clear its dependence on the parameters $d, h$, $\dot{x}_{s}$, and $\Delta u$. 
The next theorem establishes that $S_{M}^{\prime}\left(d, h, \dot{x}_{s}, \Delta u ; x\right)$ is an approximate solution of the linearized discrete equations

$$
\begin{aligned}
& k L_{j}\left(U_{j}^{n}\right) \widetilde{U}_{j}^{n} \\
& \equiv \widetilde{U}_{j}^{n+1}-\widetilde{U}_{j}^{n}+\frac{1}{2} r\left(a\left(U_{j+1}^{n}\right) \widetilde{U}_{j+1}^{n}-a\left(U_{j-1}^{n}\right) \widetilde{U}_{j-1}^{n}\right)-d\left(\widetilde{U}_{j+1}^{n}-2 \widetilde{U}_{j}^{n}+\widetilde{U}_{j-1}^{n}\right)=0,
\end{aligned}
$$

which are an approximation of the linear differential equation

$$
L_{u} \widetilde{u} \equiv \frac{\partial \widetilde{u}}{\partial t}+\frac{\partial}{\partial x}(a(u) \widetilde{u})-\frac{\partial^{2} \widetilde{u}}{\partial x^{2}}=0 .
$$

Theorem 3.4. If $S_{M}(x)$ is as defined in Theorem 3.3 , then

$$
L_{j}\left(S_{M}\left(x_{j}-\dot{x}_{s} t^{n}+X\right)\right) S_{M}^{\prime}\left(x_{j}-\dot{x}_{s} t^{n}+X\right)=o\left(h^{2 M}\right) .
$$

Proof. Using truncated Taylor series expansions, residual $N_{j}\left(S_{M}\left(x_{j}-\dot{x}_{s} t^{n}+X\right)\right)$ can be expressed as a sum of a finite number of terms, each one of the form $e_{n} h^{2 n}$ for $n \geq M+1$, with the coefficients $e_{n}$ being products of derivatives of $s(x)$ either evaluated at $x_{j}-\dot{x}_{s} t^{n}+X$ or averaged over a small interval in the neighborhood of this point, or derivatives of $f(u)$ evaluated at $s\left(x_{j}-\dot{x}_{s} t^{n}+X\right)$ or averaged over a small interval in its neighborhood.

Differentiation with respect to $X$ does not introduce any new powers of $h$, and therefore

$$
\begin{aligned}
\frac{\partial}{\partial X} N_{j}\left(S_{M}\left(x_{j}-\dot{x}_{s} t^{n}+X\right)\right) & =L_{j}\left(S_{M}\left(x_{j}-\dot{x}_{s} t^{n}+X\right)\right) S_{M}^{\prime}\left(x_{j}-\dot{x}_{s} t^{n}+X\right) \\
& =o\left(h^{2 M}\right) .
\end{aligned}
$$

3.3. Mesh-dependent viscosity. Switching to the numerical discretization with $\varepsilon=h^{\alpha}$ and $\varepsilon d$ fixed as $h \rightarrow 0$, we come to the key result which will be used in the general asymptotic analysis.

THEOREM 3.5. For constants $\dot{x}_{s}$ and $\Delta u$ satisfying the inequalities in Theorem 3.1, and with the discrete operators $N_{j}$ and $L_{j}$ as defined in (1.6) and (1.7), there exists a function $S(x)$ (which also depends on the parameters $\varepsilon d, \varepsilon^{-1} h, \dot{x}_{s}, \Delta u$ ) such that

$$
N_{j}\left(S\left(\varepsilon^{-1}\left(x_{j}-\dot{x}_{s} t^{n}\right)\right)\right)=o(\varepsilon)
$$

and

$$
L_{j}\left(S\left(\varepsilon^{-1}\left(x_{j}-\dot{x}_{s} t^{n}\right)\right)\right) S^{\prime}\left(\varepsilon^{-1}\left(x_{j}-\dot{x}_{s} t^{n}\right)\right)=o(\varepsilon),
$$

and as $x^{*} \rightarrow \infty, S\left(-x^{*}\right) \rightarrow s_{-\infty}, S\left(x^{*}\right) \rightarrow s_{\infty}=s_{-\infty}+\Delta u$, and

$$
\int_{-x^{*}}^{x^{*}} S(x) d x \rightarrow x^{*}\left(s_{-\infty}+s_{\infty}\right) .
$$

Proof. The key is to note that the finite difference equation with $\varepsilon=h^{\alpha}$ is identical to that for unit viscosity if we make the substitutions $h=\varepsilon h_{\text {unit }}, k=\varepsilon k_{\text {unit }}$.

Hence, $S_{M}\left(\varepsilon d, \varepsilon^{-1} h, \dot{x}_{s}, \Delta u ; \varepsilon^{-1}\left(x-\dot{x}_{s} t\right)\right)$ is an approximation to the discrete solution with residual error $o\left(\varepsilon^{-2 M-1} h^{2 M}\right)$. This residual error can be made $o(\varepsilon)$ as $h \rightarrow 0$ by choosing $M$ such that $(-2 M-1) \alpha+2 M>\alpha \Longleftrightarrow M>\alpha /(1-\alpha)$.

Also, for the same value of $M, S_{M}^{\prime}\left(\varepsilon d, \varepsilon^{-1} h, \dot{x}_{s}, \Delta u ; \varepsilon^{-1}\left(x-\dot{x}_{s} t\right)\right)$ is an approximate solution of the linearized discrete equation with residual error $o(\varepsilon)$. 


\section{Asymptotic approximation of discrete solutions.}

4.1. Nonlinear analysis. The numerical examples in Part 2 [GU10] consider initial data which lead to the formation of a shock after a finite time; however, the numerical analysis of such a problem would require a detailed analysis of the neighborhood of the shock formation. To simplify the analysis and keep the focus on the key aspect which is the profile of the numerical solution across the shock, we choose instead to consider first initial data $u_{0}(x)$ with a finite strength shock at $x_{s}(0)=0$. Based on this, we will be able in Part 2 [GU10] to consider more general initial data.

To be specific, the initial data is assumed to satisfy the following conditions (this will be relaxed in Part 2 [GU10]).

(A1) Apart from a discontinuity at $x_{s}(0), u_{0}(x)$ is $C^{\infty}$ with all derivatives having a finite $L_{1}$ norm over $\left(-\infty, x_{s}(0)\right)$ and $\left(x_{s}(0), \infty\right)$.

(A2) The discontinuity has finite strength for the entire time interval $[0, T]$, and no other discontinuity is formed during this time interval.

The initial discontinuity raises a new question - how to specify the initial data for the numerical discretization. The previous section has shown that if the initial data is piecewise constant, then we can expect the numerical solution to approach the traveling wave solution $S\left(\varepsilon d, \varepsilon^{-1} h, \dot{x}_{s},[u] ; \varepsilon^{-1}\left(x-\dot{x}_{s} t\right)\right)$. To avoid the requirement of performing an additional asymptotic analysis of this relaxation process, we will assume a very particular form of the initial data in the neighborhood of the initial shock. The details will be given later, but on either side of the shock the initial data is simply $U_{j}^{0}=u_{0}\left(x_{j}\right)$.

The objective now is to use a matched asymptotic analysis to construct a smooth function $V(x, t)$ such that $V_{j}^{n}=V\left(x_{j}, t^{n}\right)$ is a very close approximation to the discrete solution $U_{j}^{n}$. The stability estimate in Theorem 2.1 will be used to bound the difference $V_{j}^{n}-U_{j}^{n}$ based on the magnitude of the residual error $N_{j}\left(V_{j}^{n}\right)$.

The matched asymptotic analysis [BO78, KC81] breaks the domain into three overlapping regions:
A: $x_{s}-x>\varepsilon^{\beta}$.
B: $\left|x-x_{s}\right|<2 \varepsilon^{\beta}$.
C: $x-x_{s}>\varepsilon^{\beta}$.

Here $\beta$ is a constant just slightly less than unity so that the overlap regions contain less and less of the exponential tails of the traveling wave profile as $h \rightarrow 0$. A lower bound on $\beta$ will be determined in Theorem 4.2.

We begin with a result concerning an approximate solution in the outer region.

THEOREM 4.1. In outer regions $A$ and $C$, there exists a function $V_{o}(x, t)$, with a parametric dependence on $h$ and satisfying initial data $V_{o}(x, 0)=u_{0}(x)$, such that $N_{j}\left(V_{o}\left(x_{j}, t^{n}\right)\right)=o\left(h^{2+\alpha}\right)$.

Proof. In the outer regions, the leading order term in $V_{o}(x, t)$ is the inviscid solution $u(x, t)$ which gives a residual error whose leading order term is

$$
-\varepsilon \frac{\partial^{2} u}{\partial x^{2}} .
$$

Following the same procedure as in the proof of Theorem 3.3, this residual error can be eliminated to leading order through the addition of a correction term $\varepsilon V_{o, 1}(x, t)$ which satisfies the equation

$$
\frac{\partial V_{o, 1}}{\partial t}+\frac{\partial}{\partial x}\left(a(u) V_{o, 1}\right)=\frac{\partial^{2} u}{\partial x^{2}},
$$


subject to homogeneous initial conditions. Continuing with this process, it can be proved inductively that each correction term is $O\left(h^{m \alpha+2 n}\right)$ for integers $m, n$ with $m \geq 0, n \geq 0, m+n>0$, with the powers of $h^{2}$ arising from the second order accuracy of the central finite difference approximations. Therefore, after a finite number of steps this process gives an approximate solution $V_{o}(x, t)$ of the form

$$
V_{o}(x, t)=u(x, t)+\varepsilon V_{o, 1}(x, t)+\sum_{\alpha<m \alpha+2 n \leq \alpha+2} h^{m \alpha+2 n} V_{o, m, n}(x, t),
$$

which satisfies the initial data $V_{o}(x, 0)=u_{0}(x)$ and has a residual error which is $o\left(h^{2+\alpha}\right)$.

The next theorem considers the more difficult construction of an approximate solution in the inner region, using an inner coordinate $X \equiv \varepsilon^{-1}\left(x-x_{s}(t)\right)$. The finite difference discretization can be viewed as approximating the viscous differential equation

$$
\frac{\partial u}{\partial t}+\frac{\partial f}{\partial x}=\varepsilon \frac{\partial^{2} u}{\partial x^{2}}
$$

with $\varepsilon=h^{\alpha}$. Changing to the new inner coordinate, this equation becomes

$$
\varepsilon \frac{\partial u}{\partial t}-\dot{x}_{s}(t) \frac{\partial u}{\partial X}+\frac{\partial f}{\partial X}=\frac{\partial^{2} u}{\partial X^{2}}
$$

and small linearized perturbations of this equation due to the introduction of an inhomogeneous source term $s(x, t)$ satisfy the equation

$$
\varepsilon \frac{\partial \widetilde{u}}{\partial t}+\frac{\partial}{\partial X}\left(\left(a(u)-\dot{x}_{s}(t)\right) \widetilde{u}\right)-\frac{\partial^{2} \widetilde{u}}{\partial X^{2}}=s(x, t) .
$$

Neglecting the $O(\varepsilon)$ unsteady term, this gives the equation

$$
\frac{\partial}{\partial X}\left(\left(a(u)-\dot{x}_{s}(t)\right) \widetilde{u}\right)-\frac{\partial^{2} \widetilde{u}}{\partial X^{2}}=s(x, t),
$$

which plays a central role in the proof of the following theorem.

THEOREM 4.2. In the inner region $B$, there exists a function $V_{i}(x, t)$, with a parametric dependence on $h$, such that $N_{j}\left(V_{i}\left(x_{j}, t^{n}\right)\right)=o\left(h^{2+\alpha}\right)$, and $V_{i}(x, t)-V_{o}(x, t)=$ $o\left(h^{2+\alpha}\right)$ in the region of overlap with the outer regions $A$ and $C$, provided $\alpha>\frac{2}{3}$ and $\beta<1$ is sufficiently large.

Proof. In the overlap between regions A and B, a Taylor series expansion of $V_{o}(x, t)$ gives

$$
V_{o}(x, t)=V_{o}\left(x_{s}^{-}, t\right)+\left.\sum_{p=1}^{3} \frac{h^{p \alpha} X^{p}}{p !} \frac{\partial^{p} V_{o}}{\partial x^{p}}\right|_{\left(x_{s}^{-}(t), t\right)}+O\left(h^{4 \alpha} X^{4}\right) .
$$

Note that $V_{o}(x, t)$ itself has an asymptotic expansion in $h$ given by (4.1), and inserting this into the above will give an expansion in powers of $h^{m \alpha+2 n}$ for $m \geq 0, n \geq 0$.

A similar expression holds for the overlap between regions $\mathrm{B}$ and $\mathrm{C}$, with $x_{s}^{-}$ replaced by $x_{s}^{+}$. These form the boundary conditions for the asymptotic expansion in the inner region. To leading order, they give

$$
V_{i, 0}(X, t) \rightarrow \begin{cases}u\left(x_{s}^{-}(t), t\right), & X \rightarrow-\infty, \\ u\left(x_{s}^{+}(t), t\right), & X \rightarrow \infty,\end{cases}
$$


and hence the leading term in the asymptotic expansion in inner region $\mathrm{B}$ is

$$
V_{i, 0}(X, t)=S\left(\varepsilon d, \varepsilon^{-1} h, \dot{x}_{s}(t),[u(t)] ; X-X_{s}(t)\right),
$$

where $X_{s}(t)$ is initially an arbitrary function of time. $V_{i, 0}(X, t)$ satisfies the boundary conditions since $\dot{x}_{s}(t), u\left(x_{s}^{-}(t), t\right), u\left(x_{s}^{+}(t), t\right)$ satisfy the jump condition (1.2) which matches the jump condition (3.3) in Theorem 3.1 (with $s_{-\infty} \equiv u\left(x_{s}^{-}(t), t\right)$ and $s_{\infty} \equiv$ $u\left(x_{s}^{+}(t), t\right)$ ), which in turn is the basis for the construction of the discrete traveling wave solution in Theorem 3.5.

The nonuniqueness due to $X_{s}(t)$ in the leading order inner solution is typical of interior boundary layers (see [HY01] and page 160 in [KC81]) and is resolved through a solvability condition for a later term in the asymptotic expansion.

From (3.6) in Theorem 3.5, we have

$$
N_{j}\left(S\left(\varepsilon d, \varepsilon^{-1} h, \dot{x}_{s}, \Delta u ; \varepsilon^{-1}\left(x_{j}-\dot{x}_{s} t^{n}\right)-X_{s}\right)\right)=o(\varepsilon),
$$

when $\dot{x}_{s}, \Delta u$, and $X_{s}$ are constant, so writing

$$
V_{j}^{n}=S\left(\varepsilon d, \varepsilon^{-1} h, \dot{x}_{s}\left(t^{n}\right),\left[u\left(t^{n}\right)\right] ; \varepsilon^{-1}\left(x_{j}-x_{s}\left(t^{n}\right)\right)-X_{s}\left(t^{n}\right)\right),
$$

one obtains

$$
\begin{aligned}
& V_{j}^{n}-\frac{1}{2} r\left(f\left(V_{j+1}^{n}\right)-f\left(V_{j-1}^{n}\right)\right)+\varepsilon d\left(V_{j+1}^{n}-2 V_{j}^{n}+V_{j-1}^{n}\right) \\
& =S\left(\varepsilon d, \varepsilon^{-1} h, \dot{x}_{s}\left(t^{n}\right),\left[u\left(t^{n}\right)\right] ; \varepsilon^{-1}\left(x_{j}-x_{s}\left(t^{n}\right)-k \dot{x}_{s}\left(t^{n}\right)\right)-X_{s}\left(t^{n}\right)\right)+o(k \varepsilon) \\
& =S\left(\varepsilon d, \varepsilon^{-1} h, \dot{x}_{s}\left(t^{n}\right),\left[u\left(t^{n}\right)\right] ; \varepsilon^{-1}\left(x_{j}-x_{s}\left(t^{n+1}\right)\right)-X_{s}\left(t^{n}\right)\right)+o(k \varepsilon),
\end{aligned}
$$

since $k=o\left(\varepsilon^{2}\right)$. Hence, it follows that

$$
\begin{aligned}
& N_{j}\left(S\left(\varepsilon d, \varepsilon^{-1} h, \dot{x}_{s}\left(t^{n}\right),\left[u\left(t^{n}\right)\right] ; \varepsilon^{-1}\left(x-x_{s}\left(t^{n}\right)\right)-X_{s}\left(t^{n}\right)\right)\right) \\
& =k^{-1}\left(S\left(\varepsilon d, \varepsilon^{-1} h, \dot{x}_{s}\left(t^{n+1}\right),\left[u\left(t^{n+1}\right)\right] ; \varepsilon^{-1}\left(x_{j}-x_{s}\left(t^{n+1}\right)\right)-X_{s}\left(t^{n+1}\right)\right)\right. \\
& \left.\quad-S\left(\varepsilon d, \varepsilon^{-1} h, \dot{x}_{s}\left(t^{n}\right),\left[u\left(t^{n}\right)\right] ; \varepsilon^{-1}\left(x_{j}-x_{s}\left(t^{n+1}\right)\right)-X_{s}\left(t^{n}\right)\right)\right)+o(\varepsilon) \\
& =\left.\frac{\partial V_{i, 0}}{\partial t}\right|_{X_{j}, t^{n}}+o(\varepsilon) .
\end{aligned}
$$

Following the methodology of the proof of Theorem 3.3, this $O(1)$ residual error can be corrected by the addition of a term $\varepsilon V_{i, 1}(x, t)$ which must satisfy the equation

$$
\frac{\partial}{\partial X}\left(\left(a\left(V_{i, 0}\right)-\dot{x}_{s}\right) V_{i, 1}\right)-\frac{\partial^{2} V_{i, 1}}{\partial X^{2}}=-\frac{\partial V_{i, 0}}{\partial t},
$$

subject to the boundary conditions

$$
V_{i, 1}(X, t) \longrightarrow \begin{cases}\left.X \frac{\partial u}{\partial x}\right|_{\left(x_{s}^{-}(t), t\right)}+V_{o, 1}\left(x_{s}^{-}(t), t\right), & X \rightarrow-\infty \\ \left.X \frac{\partial u}{\partial x}\right|_{\left(x_{s}^{+}(t), t\right)}+V_{o, 1}\left(x_{s}^{+}(t), t\right), & X \rightarrow \infty .\end{cases}
$$


As with Theorem 3.2, there is a solvability condition which must be satisfied, and this is found by integrating (4.3) over the interval $\left[-X^{*}, X^{*}\right]$. Using the integral property in Theorem 3.5 , as $X^{*} \rightarrow \infty$,

$$
\int_{-X^{*}}^{X^{*}} V_{i, 0} \mathrm{~d} X \rightarrow X^{*}\left(u\left(x_{s}^{-}(t), t\right)+u\left(x_{s}^{+}(t), t\right)\right)-X_{s}(t)[u]
$$

and hence the integral of the right-hand side of (4.3) asymptotically approaches

$$
-X^{*} \frac{\mathrm{d}}{\mathrm{d} t}\left(u\left(x_{s}^{-}(t), t\right)+u\left(x_{s}^{+}(t), t\right)\right)+\frac{\mathrm{d}}{\mathrm{d} t}\left(X_{s}(t)[u]\right) .
$$

Using the boundary conditions as $X^{*} \rightarrow \infty$, the integral of the left-hand side of (4.3) becomes

$$
\begin{aligned}
{\left[\left(a\left(V_{i, 0}\right)-\dot{x}_{s}\right) V_{i, 1}-\frac{\partial V_{i, 1}}{\partial X}\right]_{-X^{*}}^{X^{*}} \longrightarrow } & {\left[\left(a(u)-\dot{x}_{s}\right) V_{o, 1}-\frac{\partial u}{\partial x}\right] } \\
& +X^{*}\left(a\left(u\left(x_{s}^{+}, t\right)\right)-\dot{x}_{s}\right) \frac{\partial u}{\partial x}\left(x_{s}^{+}, t\right) \\
& +X^{*}\left(a\left(u\left(x_{s}^{-}, t\right)\right)-\dot{x}_{s}\right) \frac{\partial u}{\partial x}\left(x_{s}^{-}, t\right) .
\end{aligned}
$$

Now, noting that

$$
\frac{\mathrm{d}}{\mathrm{d} t} u\left(x_{s}^{ \pm}(t), t\right)=-\left(a\left(u\left(x_{s}^{ \pm}, t\right)\right)-\dot{x}_{s}(t)\right) \frac{\partial u}{\partial x}\left(x_{s}^{ \pm}, t\right),
$$

it follows that the $X^{*}$ components on the two sides of the integrated equation are equal. Equating the other components gives the equation

$$
\frac{\mathrm{d}}{\mathrm{d} t}\left(X_{s}[u]\right)=\left[\left(a(u)-\dot{x}_{s}\right) V_{o, 1}-\frac{\partial u}{\partial x}\right],
$$

governing the evolution of $X_{s}(t)$ from the initial value $X_{s}(0)=0$. Equation (4.3) can now be integrated to obtain $V_{i, 1}$, with uniqueness being determined through the solvability condition for the $O\left(\varepsilon^{2}\right)$ correction term.

By continuing the asymptotic expansion and analysis, we eventually obtain an inner solution $V_{i}(x, t)$ of the form

$$
V_{i}(x, t)=V_{i, 0}(X, t)+\varepsilon V_{i, 1}(X, t)+\sum_{\alpha<m \alpha+2 n \leq \alpha+2} h^{m \alpha+2 n} V_{i, m, n}(X, t),
$$

which matches all of the terms in the asymptotic expansion of the outer solution $V_{o}$ up to and including terms proportional to $h^{2+\alpha}$, and also has a residual error which is $o\left(h^{2+\alpha}\right)$.

In the overlap region, the exponential tails of the traveling wave solution behave like $\exp (-c|X|)$ for some constant $c$. Since $|X|=O\left(\varepsilon^{\beta-1}\right)=O\left(h^{-\alpha(1-\beta)}\right)$, these exponential tails are $o\left(h^{q}\right) \forall q>0$. Furthermore, noting that $4 \alpha>2+\alpha$ because of the lower bound on $\alpha$, let $\gamma$, satisfying the inequalities $4 \alpha \geq \gamma>2+\alpha$, be the lowest power of $h$ for which the corresponding term in the asymptotic expansion of the outer solution given at the beginning of this proof does not have a matching counterpart in the inner expansion. In this case, at worst the mismatch between the inner and outer 
solutions in the overlap region is $O\left(h^{\gamma} X^{4}\right)=O\left(h^{\gamma-4 \alpha(1-\beta)}\right)$. Provided $\beta$ satisfies the lower bound given by the condition

$$
\gamma-4 \alpha(1-\beta)>2+\alpha \quad \Longrightarrow \quad \beta>1-\frac{\gamma-2-\alpha}{4 \alpha},
$$

it then follows that the inner and outer approximate solutions match to within $O\left(h^{2+\alpha}\right)$.

We can now combine these approximate solutions to form a patched solution which gives an accurate approximation of a discrete solution.

THEOREM 4.3. There exists a function $V(x, t)$ with parametric dependence on $h$, and initial data $U_{j}^{0} \equiv V\left(x_{j}, 0\right)$ producing the numerical solution $U_{j}^{n}$, such that $\left\|U_{j}^{0}-u_{0}\left(x_{j}\right)\right\|_{1}=O\left(h^{\alpha}\right)$ and $\left\|U_{j}^{n}-V\left(x_{j}, t^{n}\right)\right\|_{1}=o\left(h^{2+\alpha}\right)$.

Proof. The patched solution $V(x, t)$ is defined using a $C^{\infty}$ blending function $P(x)$ which has constant value $P(x)=1$ for $|x|<1$ and $P(x)=0$ for $|x|>2$. Using this, we define $V(x, t)$ through

$$
\begin{aligned}
V(x, t) & =V_{i}(x, t)+\left(1-P\left(\varepsilon^{-\beta}\left(x-x_{s}(t)\right)\right)\right)\left(V_{o}(x, t)-V_{i}(x, t)\right) \\
& =V_{o}(x, t)+P\left(\varepsilon^{-\beta}\left(x-x_{s}(t)\right)\right)\left(V_{i}(x, t)-V_{o}(x, t)\right) .
\end{aligned}
$$

Setting $V_{j}^{n}=V\left(x_{j}, t^{n}\right)$, the residual error $N_{j}\left(V_{j}^{n}\right)$ is $o\left(h^{2+\alpha}\right)$ outside the overlap regions. Within the overlap region, defining $P_{j}^{n}=P\left(\varepsilon^{-\beta}\left(x_{j}-x_{s}\left(t^{n}\right)\right)\right)$ and $\Delta V_{j}^{n}=$ $V_{i}\left(x_{j}, t^{n}\right)-V_{o}\left(x_{j}, t^{n}\right)$, it can be verified that

$$
\begin{aligned}
N_{j}\left(V_{j}^{n}\right) & =P_{j}^{n} N_{j}\left(V_{i, j}^{n}\right)+\left(1-P_{j}^{n}\right) N_{j}\left(V_{o, j}^{n}\right) \\
& +\frac{1}{k}\left(P_{j}^{n+1}-P_{j}^{n}\right) \Delta V_{j}^{n+1} \\
& +\frac{1}{2 h}\left(P_{j+1}^{n}-P_{j}^{n}\right)\left[f\left(V_{i, j+1}^{n}\right)-f\left(V_{o, j+1}^{n}\right)\right] \\
& +\frac{1}{2 h}\left(P_{j}^{n}-P_{j-1}^{n}\right)\left[f\left(V_{i, j-1}^{n}\right)-f\left(V_{o, j-1}^{n}\right)\right] \\
& +\frac{1}{2 h}\left[f\left(V_{j+1}^{n}\right)-P_{j+1}^{n} f\left(V_{i, j+1}^{n}\right)-\left(1-P_{j+1}^{n}\right) f\left(V_{o, j+1}^{n}\right)\right] \\
& -\frac{1}{2 h}\left[f\left(V_{j-1}^{n}\right)-P_{j-1}^{n} f\left(V_{i, j-1}^{n}\right)-\left(1-P_{j-1}^{n}\right) f\left(V_{o, j-1}^{n}\right)\right] \\
& -\frac{\varepsilon}{h^{2}}\left(P_{j+1}^{n}-2 P_{j}^{n}+P_{j-1}^{n}\right) \Delta V_{j}^{n} \\
& -\frac{\varepsilon}{h^{2}}\left[\left(P_{j+1}^{n}-P_{j}^{n}\right)\left(\Delta V_{j+1}^{n}-\Delta V_{j}^{n}\right)+\left(P_{j}^{n}-P_{j-1}^{n}\right)\left(\Delta V_{j}^{n}-\Delta V_{j-1}^{n}\right)\right] .
\end{aligned}
$$

On line 1 of this equation, the linear interpolation of the residual errors is $o\left(h^{2+\alpha}\right)$. Since $k^{-1}\left(P_{j}^{n+1}-P_{j}^{n}\right)=O\left(\varepsilon^{-\beta}\right)$ and $\Delta V=o\left(h^{2+\alpha}\right)$, the term on line 2 is $o\left(h^{-\alpha \beta+2+\alpha}\right)$. Similarly, the terms on lines 3 and 4 are also $o\left(h^{-\alpha \beta+2+\alpha}\right)$. Due to standard quadratic error bounds for linear interpolation, the terms on lines 5 and 6 are $o\left(h^{-1+2(2+\alpha)}\right)$. The terms on lines 7 and 8 are $o\left(h^{\alpha-2 \alpha \beta+2+\alpha}\right)$, since each derivative of $P$ and $\Delta V$ introduces a factor $h^{-\alpha \beta}$. Since the overlap regions have measure $O\left(h^{\alpha \beta}\right)$, it follows that the $l_{1}$ norm of the residual on the overlap regions is $o\left(h^{2+\alpha}\right)$.

From the above, plus assumption (A1) which gives a finite bound for the $L_{1}$ norm of the derivatives in the residual, we conclude that $\left\|N_{j}\left(V_{j}^{n}\right)\right\|_{1}=o\left(h^{2+\alpha}\right)$. Hence, 
since $U_{j}^{n}$ is the numerical solution corresponding to initial data $U_{j}^{0}=V_{j}^{0}$, then by the stability estimate in Theorem 2.1 it follows that

$$
\left\|U_{j}^{n}-V_{j}^{n}\right\|_{1}=o\left(h^{2+\alpha}\right)
$$

4.2. Linear analysis. We now construct a matched asymptotic approximation to the numerical solution of the linear discrete equations subject to the initial data $\widetilde{U}_{j}^{0}=\widetilde{u}_{0}\left(x_{j}\right)$, where throughout this subsection $\widetilde{u}_{0}$ is $C^{\infty}$.

THEOREM 4.4. In outer regions $A$ and $C$, there exists a function $\widetilde{V}_{o}(x, t)$, with parametric dependence on $h$, satisfying initial data $\widetilde{V}_{o}(x, 0)=\widetilde{u}_{0}(x)$, such that

$$
L_{j}\left(V_{o}\left(x_{j}, t^{n}\right)\right) \widetilde{V}_{o}\left(x_{j}, t^{n}\right)=o(\varepsilon) .
$$

Proof. As with the nonlinear analysis, the leading order term in the asymptotic expansion for the linear solution is the analytic solution $\widetilde{u}(x, t)$ which gives a residual error which is $O(\varepsilon)$. By matching all residual error terms which are $O(\varepsilon)$, including those coming from the asymptotic expansion of the nonlinear discrete solution, this can be compensated for through the addition of a correction $\varepsilon \widetilde{V}_{o, 1}(x, t)$ satisfying the equation

$$
\frac{\partial \widetilde{V}_{o, 1}}{\partial t}+\frac{\partial}{\partial x}\left(a(u) \widetilde{V}_{o, 1}\right)=\frac{\partial^{2} \widetilde{u}}{\partial x^{2}}-\frac{\partial}{\partial x}\left(a^{\prime}(u) V_{o, 1} \widetilde{V}_{o, 0}\right),
$$

subject to homogeneous initial data. Setting $\widetilde{V}_{o}(x, t)=\widetilde{u}(x, t)+\varepsilon \widetilde{V}_{o, 1}(x, t)$ then gives an outer solution with the required properties.

THEOREM 4.5. In the inner region $B$, there exists a function $\widetilde{V}_{i}(x, t)$, with parametric dependence on $h$, such that $L_{j}\left(V_{i}\left(x_{j}, t^{n}\right)\right) \widetilde{V}_{i}\left(x_{j}, t^{n}\right)=O(\varepsilon)$ and $\widetilde{V}_{i}(x, t)-$ $\widetilde{V}_{o}(x, t)=o(\varepsilon)$ in the region of overlap with the outer regions $A$ and $C$.

Proof. Because the shock width is $O(\varepsilon)$, a linearized displacement of the shock of unit magnitude corresponds to an $O\left(\varepsilon^{-1}\right)$ linear solution. Accordingly, in region B, the leading term in the asymptotic expansion is

$$
\begin{aligned}
\varepsilon^{-1} \widetilde{V}_{i,-1}(x, t) & =-\widetilde{X}_{s}(t) \frac{\partial V_{i, 0}}{\partial x} \\
& =-\varepsilon^{-1} \widetilde{X}_{s}(t) S^{\prime}\left(\dot{x}_{s}(t),[u(t)] ; \varepsilon^{-1}\left(x-x_{s}(t)\right)-X_{s}(t)\right)
\end{aligned}
$$

for some as yet undetermined function $\widetilde{X}_{s}(t)$, and with the parametric dependence of $S^{\prime}$ on the constants $\varepsilon d, \varepsilon^{-1} h$ omitted for brevity.

Since, from (3.7) in Theorem 3.5,

$$
L_{j}\left(S\left(\dot{x}_{s}, \Delta u ; \varepsilon^{-1}\left(x_{j}-\dot{x}_{s} t^{n}\right)-X_{s}\right)\right) S^{\prime}\left(\dot{x}_{s}, \Delta u ; \varepsilon^{-1}\left(x_{j}-\dot{x}_{s} t^{n}\right)-X_{s}\right)=o(\varepsilon)
$$

when $\dot{x}_{s}, \Delta u$, and $X_{s}$ are constant, it follows that

$$
\begin{aligned}
L_{j} & \left(V_{i, 0}\left(x_{j}, t^{n}\right)+\varepsilon V_{i, 1}\left(x_{j}, t^{n}\right)\right) \widetilde{V}_{i,-1}\left(x_{j}, t^{n}\right) \\
= & -k^{-1}\left(\widetilde{X}_{s}\left(t^{n+1}\right) S^{\prime}\left(\dot{x}_{s}\left(t^{n+1}\right),\left[u\left(t^{n+1}\right)\right] ; \varepsilon^{-1}\left(x_{j}-x_{s}\left(t^{n+1}\right)\right)-X_{s}\left(t^{n+1}\right)\right)\right. \\
& \left.-t X\left(t^{n}\right) S^{\prime}\left(\dot{x}_{s}\left(t^{n}\right),\left[u\left(t^{n}\right)\right] ; \varepsilon^{-1}\left(x_{j}-x_{s}\left(t^{n+1}\right)\right)-X_{s}\left(t^{n}\right)\right)\right) \\
& +\varepsilon \frac{\partial}{\partial x}\left(a^{\prime}\left(V_{i, 0}\left(x_{j}, t^{n}\right)\right) V_{i, 1}\left(x_{j}, t^{n}\right) \widetilde{V}_{i,-1}\left(x_{j}, t^{n}\right)\right)+o(\varepsilon) \\
= & \frac{\partial}{\partial t} \widetilde{V}_{i,-1}\left(X_{j}, t^{n}\right)+\frac{\partial}{\partial X}\left(a^{\prime}\left(V_{i, 0}\left(x_{j}, t^{n}\right)\right) V_{i, 1}\left(x_{j}, t^{n}\right) \widetilde{V}_{i,-1}\left(x_{j}, t^{n}\right)\right)+o(\varepsilon) .
\end{aligned}
$$


This $O(1)$ residual error can be corrected by the addition of a term $\widetilde{V}_{i, 0}(x, t)$ which must satisfy (see (4.2))

$$
\frac{\partial}{\partial X}\left(\left(a\left(V_{i, 0}\right)-\dot{x}_{s}\right) \widetilde{V}_{i, 0}\right)-\frac{\partial^{2} \widetilde{V}_{i, 0}}{\partial X^{2}}=-\frac{\partial \widetilde{V}_{i,-1}}{\partial t}-\frac{\partial}{\partial X}\left(a^{\prime}\left(V_{i, 0}\right) V_{i, 1} \widetilde{V}_{i,-1}\right),
$$

subject to the boundary conditions

$$
\widetilde{V}_{i, 0}(X, t) \rightarrow\left\{\begin{array}{l}
\widetilde{u}\left(x_{s}^{-}(t), t\right), \quad X \rightarrow-\infty, \\
\widetilde{u}\left(x_{s}^{+}(t), t\right), \quad X \rightarrow \infty .
\end{array}\right.
$$

Again, as in Theorem 3.2, there is a solvability condition which is found by integrating over an interval $\left[-X^{*}, X^{*}\right]$, and then taking the limit $X^{*} \rightarrow \infty$ to obtain

$$
\frac{\mathrm{d}}{\mathrm{d} t}\left(\widetilde{X}_{s}[u]\right)=\left[\left(a(u)-\dot{x}_{s}\right) \widetilde{u}_{o, 0}\right]
$$

subject to initial condition $\widetilde{X}_{s}(0)=0$. This shows that $\widetilde{X}_{s}(t)$ satisfies the same equation as the inviscid linearized shock displacement $\widetilde{x}_{s}(t),(1.4)$, and so is identical to it.

Continuing in this way with a further level of additional correction, we obtain an inner solution $\widetilde{V}_{i}(x, t)$ of the form

$$
\widetilde{V}_{i}(x, t)=\varepsilon^{-1} \widetilde{V}_{i,-1}(X, t)+\widetilde{V}_{i, 0}(X, t)+\varepsilon \widetilde{V}_{i, 1}(X, t)
$$

which has a residual error which is $O(\varepsilon)$, and whose value and derivatives match the outer solution in the overlap regions to within $o(\varepsilon)$.

We can now construct a patched asymptotic approximation $\widetilde{V}(x, t)$ over the whole interval.

TheOREM 4.6. Given the nonlinear initial conditions $U_{j}^{0}$ as defined in Theorem 4.3 , there exists a function $\widetilde{V}(x, t)$ such that $\left\|\widetilde{U}_{j}^{n}-\widetilde{V}\left(x_{j}, t^{n}\right)\right\|_{1}=o(\varepsilon)$.

Proof. $\widetilde{V}(x, t)$ is defined using the same blending function as in the proof of Theorem 4.3 to give

$$
\begin{aligned}
\widetilde{V}(x, t) & =\widetilde{V}_{i}(X, t)+\left(1-P\left(\varepsilon^{-\beta}\left(x-x_{s}(t)\right)\right)\right)\left(\widetilde{V}_{o}(x, t)-\widetilde{V}_{i}(X, t)\right) \\
& =\widetilde{V}_{o}(x, t)+P\left(\varepsilon^{-\beta}\left(x-x_{s}(t)\right)\right)\left(\widetilde{V}_{i}(x, t)-\widetilde{V}_{o}(X, t)\right) .
\end{aligned}
$$

Setting $\widetilde{V}_{j}^{n}=\widetilde{V}\left(x_{j}, t^{n}\right)$, following the same approach as in the proof of Theorem 4.3 it can be proved that the $l_{1}$ norm of the residual error $L_{j}\left(V_{j}^{n}\right) \widetilde{V}_{j}^{n}$ is $o(\varepsilon)$. Hence, combining the stability estimate in Theorem 2.2 and the result in Theorem 4.3, we obtain

$$
\left\|\widetilde{U}_{j}^{n}-\widetilde{V}_{j}^{n}\right\|_{1}=o(\varepsilon) .
$$

5. Functional errors. We now come to the main theorem of the paper, proving the convergence of the numerical approximation to the linear functional.

THEOREM 5.1. If $U_{j}^{n}$ satisfies the nonlinear discrete equations subject to the initial data $U_{j}^{0}$ specified in Theorem 4.3, and $\widetilde{U}_{j}^{n}$ satisfies the linear discrete equations with initial data $\widetilde{U}_{j}^{0}=\widetilde{u}_{0}\left(x_{j}\right)$, and if $\widetilde{J}$ and $\widetilde{J}_{h}$ are as defined in the introduction, then $\left|\widetilde{J}_{h}-\widetilde{J}\right|=O(\varepsilon)$. 
Proof. Taking $V_{j}^{n}$ and $\widetilde{V}_{j}^{n}$ to be as defined in Theorems 4.3 and 4.6, the difference between the true value of the linear functional

$$
\begin{aligned}
\widetilde{J}= & \int_{-\infty}^{x_{s}(T)} \gamma(x) G^{\prime}(u(x, T)) \widetilde{u}(x, T) \mathrm{d} x-\widetilde{x}_{s}(T) \gamma\left(x_{s}(T)\right)[G]_{T} \\
& +\int_{x_{s}(T)}^{\infty} \gamma(x) G^{\prime}(u(x, T)) \widetilde{u}(x, T) \mathrm{d} x
\end{aligned}
$$

and the discrete approximation

$$
\widetilde{J}_{h}=h \sum_{j} \gamma\left(x_{j}\right) G^{\prime}\left(U_{j}^{N}\right) \widetilde{U}_{j}^{N}
$$

can be bounded by the sum of three terms:

$$
\begin{aligned}
\left|\widetilde{J}_{h}-\widetilde{J}\right| & \leq\left|h \sum_{j} \gamma\left(x_{j}\right)\left(G^{\prime}\left(U_{j}^{N}\right) \widetilde{U}_{j}^{N}-G^{\prime}\left(V_{j}^{N}\right) \widetilde{V}_{j}^{N}\right)\right| \\
& +\left|h \sum_{j} \gamma\left(x_{j}\right) G^{\prime}\left(V_{j}^{N}\right) \widetilde{V}_{j}^{N}-\int_{-\infty}^{\infty} \gamma(x) G^{\prime}(V(x, T)) \widetilde{V}(x, T) \mathrm{d} x\right| \\
& +\left|\int_{-\infty}^{\infty} \gamma(x) G^{\prime}(V(x, T)) \widetilde{V}(x, T) \mathrm{d} x-\widetilde{J}\right| .
\end{aligned}
$$

(i) Considering the first term,

$$
G^{\prime}\left(U_{j}^{N}\right) \widetilde{U}_{j}^{N}-G^{\prime}\left(V_{j}^{N}\right) \widetilde{V}_{j}^{N}=G^{\prime}\left(U_{j}^{N}\right)\left(\widetilde{U}_{j}^{N}-\widetilde{V}_{j}^{N}\right)+\left(G^{\prime}\left(U_{j}^{N}\right)-G^{\prime}\left(V_{j}^{N}\right)\right) \widetilde{V}_{j}^{N},
$$

and hence

$$
\begin{aligned}
& \left|h \sum_{j} \gamma\left(x_{j}\right)\left(G^{\prime}\left(U_{j}^{N}\right) \widetilde{U}_{j}^{N}-G^{\prime}\left(V_{j}^{N}\right) \widetilde{V}_{j}^{N}\right)\right| \\
& \quad \leq\|\gamma\|_{\infty}\left(g_{1}\left\|\widetilde{U}^{N}-\widetilde{V}^{N}\right\|_{1}+g_{2}\left\|U^{N}-V^{N}\right\|_{1}\left\|\widetilde{V}^{N}\right\|_{\infty}\right),
\end{aligned}
$$

where

$$
g_{1}=\sup _{|v| \leq\|u\|_{\infty}} G^{\prime}(v), \quad g_{2}=\sup _{|v| \leq\|u\|_{\infty}} G^{\prime \prime}(v) .
$$

Theorems 4.3, 4.5, and 4.6 give $\left\|\widetilde{U}^{N}-\widetilde{V}^{N}\right\|_{1}=o(\varepsilon),\left\|U^{N}-V^{N}\right\|_{1}=o\left(h^{2+\alpha}\right)=$ $o\left(h^{2} \varepsilon\right)$, and $\left\|\widetilde{V}^{N}\right\|_{\infty}=O\left(\varepsilon^{-1}\right)$, and hence this first term is $o(\varepsilon)$.

(ii) Since $V_{j}^{N}=V\left(x_{j}, T\right)$ and $\widetilde{V}_{j}^{N}=\widetilde{V}\left(x_{j}, T\right)$, the second term corresponds to the error in using trapezoidal integration to approximate the integral of

$$
f(x) \equiv \gamma(x) G^{\prime}(V(x, T)) \widetilde{V}(x, T) .
$$

$V(x, T)$ and $\widetilde{V}(x, T)$ are both smooth, so the error is bounded by the Euler-Maclaurin error formula [SB80]

$$
\left|h \sum_{j} f\left(x_{j}\right)-\int_{-\infty}^{\infty} f(x) \mathrm{d} x\right| \leq \frac{h^{2 M}}{(2 M) !} \int_{-\infty}^{\infty}\left|\bar{B}_{2 m}\left(\frac{x}{h}\right) f^{(2 M)}\right| \mathrm{d} x
$$


for any integer $M$, with $\bar{B}_{2 m}(x)$ being the periodic extension of Bernoulli polynomials on $[0,1][\mathrm{SB} 80]$. Since $\left\|f^{(2 M)}\right\|_{\infty}=O\left(h^{-(2 M+1) \alpha}\right)$, it is possible to choose $M$ sufficiently large so that $2 M-(2 M+1) \alpha>\alpha$, and hence this second term is $o(\varepsilon)$.

(iii) For the third term, we start by evaluating the leading order behavior of the integral in the inner region:

$$
\begin{aligned}
& \int_{B} \gamma(x) G^{\prime}(V(x, T)) \tilde{V}(x, T) \mathrm{d} x \\
& =\int_{B} \gamma(x) G^{\prime}\left(V_{i, 0}(x, T)+\varepsilon V_{i, 1}(x, T)\right)\left(-\widetilde{x}_{s} \frac{\partial V_{i, 0}}{\partial x}(x, T)+\widetilde{V}_{i, 0}(x, T)\right) \mathrm{d} x+o(\varepsilon) \\
& =-\widetilde{x}_{s} \gamma\left(x_{s}(T)\right) \int_{B} G^{\prime}\left(V_{i, 0}(x, T)\right) \frac{\partial V_{i, 0}}{\partial x}(x, T) \mathrm{d} x \\
& \quad-\varepsilon \widetilde{x}_{s} \int_{B} \gamma(x) G^{\prime \prime}\left(V_{i, 0}(x, T)\right) V_{i, 1}(x, T) \frac{\partial V_{i, 0}}{\partial x}(x, T) \mathrm{d} x \\
& \quad-\widetilde{x}_{s} \int_{B}\left(\gamma(x)-\gamma\left(x_{s}(T)\right)\right) G^{\prime}\left(V_{i, 0}(x, T)\right) \frac{\partial V_{i, 0}}{\partial x}(x, T) \mathrm{d} x \\
& \quad+\int_{B} \gamma(x) G^{\prime}\left(V_{i, 0}(x, T)\right) \widetilde{V}_{i, 0}(x, T) \mathrm{d} x+o(\varepsilon) .
\end{aligned}
$$

In this final expression, the first integral gives $[G(u)]_{T}$. The second integral is $O(1)$ since $\frac{\partial V_{i, 0}}{\partial x}(x, T)=O\left(\varepsilon^{-1}\right)$ in the innermost region of size $O(\varepsilon)$ and tails off exponentially outside this, and hence $\left\|\frac{\partial V_{i, 0}}{\partial x}(x, T)\right\|_{1}=O(1)$. The third integral is $O(\varepsilon)$ since the product $\left(\gamma(x)-\gamma\left(x_{s}(T)\right)\right) \frac{\partial V_{i, 0}}{\partial x}(x, T)$ is $O(1)$ in the innermost region and tails off exponentially. Finally, noting that $G^{\prime}\left(V_{i, 0}(x, T)\right)-G^{\prime}(u(x, T))=O(1)$ and $\widetilde{V}_{i, 0}(x, T)-\widetilde{u}_{o, 0}(x, T)=O(1)$ uniformly in the innermost region and tail off exponentially to an $O\left(\varepsilon^{\beta}\right)$ value outside this, the fourth integral is equal to

$$
\int_{B} \gamma(x) G^{\prime}(u(x, T)) \widetilde{u}_{o, 0} \mathrm{~d} x+O(\varepsilon) .
$$

Adding the contributions from the outer regions then gives

$$
\begin{aligned}
& \int_{-\infty}^{\infty} \gamma(x) G^{\prime}(V(x, T)) \widetilde{V}(x, T) \mathrm{d} x \\
& =-\widetilde{x}_{s} \gamma\left(x_{s}(T)\right)[G(u)]_{T}+\int_{-\infty}^{\infty} \gamma(x) G^{\prime}(u(x, T)) \widetilde{u}_{o, 0}(x, T) \mathrm{d} x+O(\varepsilon) \\
& =\widetilde{J}+O(\varepsilon) .
\end{aligned}
$$

Hence the third term is $O(\varepsilon)$, giving the dominant contribution of the three terms.

This concludes the proof that the error in the discrete approximation to the linearized functional is $O(\varepsilon)$, as it would be for a model problem without a shock.

6. Conclusions. This paper has analyzed the convergence of approximate linear solutions for a class of convex flux functions using a particular modified Lax-Friedrichs discretization. It has been proved that in the case of a single shock, the linear solution converges pointwise everywhere except at the shock, and the shock itself is treated correctly in the sense that the value of integral output functionals also converges. 
The proofs rely upon the facts that (1) the linear discretization is a linearization of the nonlinear discretization; and (2) the number of mesh points across the smeared shock increases as $h \rightarrow 0$.

In Part 2 [GU10] we will continue our analysis and present numerical results that confirm our analytical findings. Moreover, we will show that the results of the present paper hold also for Dirac initial data for the linearized equation. From this we deduce that the adjoint approximation also converges pointwise everywhere except along the two characteristics at which it is discontinuous. Finally, the convergence of the adjoint solution will be extended to cases with multiple shocks.

The modified Lax-Friedrichs discretization which is analyzed in this paper is not a great choice as a practical numerical method, since it provides only $O\left(h^{\alpha}\right)$ convergence for $0<\alpha<1$. A better numerical method would use adaptive smoothing, reducing the magnitude of $\varepsilon$ or switching to a fourth difference smoothing in the smooth regions on either side of the shocks, together with adaptive grid resolution to reduce the magnitude of the grid spacing $h$ in the vicinity of the shock. Thus, the contribution of this paper and Part 2 [GU10] is to prove convergence of a simplified discretization, in order to provide insight and guidance to those trying to construct more accurate, practical methods.

\section{REFERENCES}

[AV99] W. K. Anderson And V. Venkatakrishnan, Aerodynamic design optimization on unstructured grids with a continuous adjoint formulation, Comput. \& Fluids, 28 (1999), pp. 443-480.

[BD03] T. BARTh AND H. DECONINCK, EDs., Error Estimation and Adaptive Discretization Methods in Computational Fluid Dynamics, Lect. Notes Comput. Sci. Eng. 25, Springer-Verlag, Berlin, 2003.

[BJ98] F. Bouchut AND F. JAMES, One-dimensional transport equations with discontinuous coefficients, Nonlinear Anal., 32 (1998), pp. 891-933.

[BO78] C. M. Bender And S. A. Orszag, Advanced Mathematical Methods for Scientists and Engineers, McGraw-Hill, New York, 1978.

[BP03] C. Bardos And O. Pironneau, Derivatives and control in the presence of shocks, Comput. Fluid Dynam. J., 11 (2003), pp. 383-392.

[BR01] R. BECKER AND R. RANNACHER, An optimal control approach to error control and mesh adaptation, in Acta Numerica 2001, A. Iserles, ed., Cambridge University Press, Cambridge, UK, 2001, pp. 1-102.

[CM80] M. CRANDAll AND A. MAJDA, Monotone difference approximations for scalar conservation laws, Math. Comp., 34 (1980), pp. 1-21.

[CT87] P. Courtier And O. TAlagrand, Variational assimilation of meteorological observations with the adjoint vorticity equation, II, Numerical results, Q. J. R. Meteorol. Soc., 113 (1987), pp. 1329-1347.

[DMlm95] G. Dal Maso, P. G. Lefloch, And F. Murat, Definition and weak stability of nonconservative products, J. Math. Pures Appl. (9), 74 (1995), pp. 483-548.

[Gil96] M. B. GiLES, Analysis of the accuracy of shock-capturing in the steady quasi-1D Euler equations, Internat. J. Comput. Fluid Dynam., 5 (1996), pp. 247-258.

[Gil03] M. B. Giles, Discrete adjoint approximations with shocks, in Hyperbolic Problems: Theory, Numerics, Applications, T. Hou and E. Tadmor, eds., Springer-Verlag, New York, 2003.

[GP98] M. B. Giles AND N. A. Pience, On the properties of solutions of the adjoint Euler equations, in Numerical Methods for Fluid Dynamics VI, M. Baines, ed., ICFD, Reading, UK, 1998.

[GP01] M. B. GILES AND N. A. PIERCE, Analytic adjoint solutions for the quasi-onedimensional Euler equations, J. Fluid Mech., 426 (2001), pp. 327-345.

[GU10] M. GILES AND S. Ulbrich, Convergence of linearized and adjoint approximations for discontinuous solutions of conservation laws. Part 2: Adjoint approximations and extensions, SIAM J. Numer. Anal., 48 (2010), pp. 905-921. 
[HCl94] K. C. Hall, W. S. Clark, and C. B. Lorence, A linearized Euler analysis of unsteady transonic flows in turbomachinery, J. Turbomachinery, 116 (1994), pp. 477488.

[HL94] T. Y. Hou AND P. G. LEFLOCH, Why nonconservative schemes converge to wrong solutions: Error analysis, Math. Comp., 62 (1994), pp. 497-530.

[HN03] C. Homescu And I. M. NAvon, Optimal control of flow with discontinuities, J. Comput. Phys., 187 (2003), pp. 660-682.

[HY01] S. I. Hariharan AND G. W. Young, Comparison of asymptotic solutions of a phasefield model to a sharp-interface model, SIAM J. Appl. Math., 62 (2001), pp. 244263.

[Jam95] A. JAmeson, Optimum aerodynamic design using control theory, in Computational Fluid Dynamics Review 1995, M. Hafez and K. Oshima, eds., John Wiley \& Sons, New York, 1995, pp. 495-528.

[KC81] J. Kevorkian and J. D. Cole, Perturbation Methods in Applied Mathematics, Appl. Math. Sci. 34, Springer-Verlag, New York, 1981.

[LeF90] P. LEFLOCH, An existence and uniqueness result for two nonstrictly hyperbolic systems, in Nonlinear Evolution Equations That Change Type, IMA Vol. Math. Appl. 27, Springer-Verlag, New York, 1990, pp. 126-138.

[LG94] D. R. Lindquist AND M. B. Giles, Validity of linearized unsteady Euler equations with shock capturing, AIAA J., 32 (1994), pp. 46-53.

[NT92] H. Nessyahu AND E. TADMOR, The convergence rate of approximate solutions for nonlinear scalar conservation laws, SIAM J. Numer. Anal., 29 (1992), pp. 15051519.

[SB80] J. Stoen And R. Bulinsch, Introduction to Numerical Analysis, Springer-Verlag, New York, 1980.

[SW98] K. SReenivas And D. L. Whitfield, Time- and frequency-domain numerical simulation of linearized Euler equations, AIAA J., 36 (1998), pp. 968-975.

[Tad91] E. TADMOR, Local error estimates for discontinuous solutions of nonlinear hyperbolic equations, SIAM J. Numer. Anal., 28 (1991), pp. 891-906.

[TC87] O. TAlaGRAND AND P. Courtier, Variational assimilation of meteorological observations with the adjoint vorticity equation, I, Theory, Q. J. R. Meteorol. Soc., 113 (1987), pp. 1311-1328.

[Ulb02] S. ULBRICH, A sensitivity and adjoint calculus for discontinuous solutions of hyperbolic conservation laws with source terms, SIAM J. Control Optim., 41 (2002), pp. 740797.

[Ulb03] S. UlBRICH, Adjoint-based derivative computations for the optimal control of discontinuous solutions of hyperbolic conservation laws, Systems Control Lett., 48 (2003), pp. 313-328. 